\title{
Detection and characterization of multidrug resistance and extended-spectrum-beta-lactamase-producing (ESBLs) Pseudomonas aeruginosa isolates in teaching hospital
}

\author{
Zahra Tavajjohi $^{1}$, Rezvan Moniri ${ }^{2 \star}$ and Ahmad Khorshidi ${ }^{2}$ \\ ${ }^{1}$ Department of Biology, Faculty of Science, Alzahra University, Tehran, Iran. \\ ${ }^{2}$ Kashan University of Medical Sciences, Kashan, Iran.
}

\begin{abstract}
Accepted 30 July, 2011
Pseudomonas aeruginosa is the most common pathogen causing nosocomial infections. The objective of this study was to investigate the extended-spectrum-beta-lactamase (ESBLs) producing and multidrug resistance of hospital isolates of $P$. aeruginosa and to determine the presence of several resistance genes. A total of 86 isolates of $P$. aeruginosa were collected from teaching hospital in Kashan, Iran. Susceptibility to eight antimicrobial agents was performed by disk diffusion method. ESBL-phenotypic detection was carried out by double-disk synergy test; and the presence of the genes encoding of bla(TEM), bla(SHV), bla(CTX-M), bla(OXA) and bla(GES)-like genes was studied by polymerase chain reaction. The prevalence of ESBLs was $8.1 \%$. The presence of genes encoding ESBLs was confirmed in seven isolates, comprising seven bla(GES-2), one bla(SHV-1), one bla(SHV-5) and one bla(CTX-M-1) genes. $P$. aeruginosa demonstrated the highest resistance rate to piperacillin (38.4\%), $67.5 \%$ of isolates were sensitive to imipenem whereas $32.5 \%$ were MDR (resistant to three or more classes of antibiotics). A multidrug-resistant (MDR) phenotype occurred frequently in $P$. aeruginosa. bla(GES-2), which compromises the efficacy of imipenem detected in all of seven ESBLproducing $P$. aeruginosa strains. Proper infection control practices and barriers are essential to prevent spreading and outbreaks of ESBL-producing and MDR $P$. aeruginosa in our teaching hospital.
\end{abstract}

Key words: Pseudomonas aeruginosa, extended-spectrum-beta-lactamase, multidrug-resistant.

\section{INTRODUCTION}

Pseudomonas aeruginosa is widely known as an opportunistic organism, frequently involved in infections of immunosuppressed patients, and also causes outbreaks of hospital-acquired infections (Wirth et al., 2009). P. aeruginosa is intrinsically resistant to many antimicrobial agents, including most $\beta$-lactams, the older quinolones, chloramphenicol, tetracycline, macrolides, trimethoprimsulfamethoxazole and rifampin (Dundar and Otkun, 2010; Rossolini and Mantengoli, 2005). Multidrug resistance can be relatively common amongst nosocomial isolates of $P$. aeruginosa, which represent the large majority of

*Corresponding author. E-mail: moniri@kaums.ac.ir. Tel: + 98 $3615550021-25(539)$. Fax: + 983615551112. clinical isolates (Rossolini and Mantengoli, 2005). Resistance in $P$. aeruginosa may be mediated via several distinct mechanisms including the production of $\beta$ lactamases, efflux pumps, and target-site or outer membrane modifi-cations (Tam et al., 2010). The production of extended-spectrum beta-lactamases (ESBLs) confers resistance at various levels to expandedspectrum cephalosporins, such as cefotaxime and ceftazidime, and to aztreonam, but not normally to the cephamycins and carbapenems (Mirsalehian et al., 2010). These enzymes are encoded by different genes located on either chromosomes or plasmids (Chuang and Rasmussen, 2006). Two major classification schemes exist for categorizing $\beta$-lactamase enzymes: Ambler classes $A$ through $D$, based on amino acid sequence homology, and Bush-Jacoby-Medeiros groups 1 through 
4, based on substrate and inhibitor profile (Drawz et al., 2010). The activity of Class A enzymes is inhibited in vitro by $\beta$-lactamase inhibitors such as clavulanic acid, sulbactam and tazobactam but those belonging to class $D$ are not (Bradford, 2001). Recently, however, several class $A, B$ and $D$ extended spectrum $\beta$-lactamases have been reported in $P$. aeruginosa (Park et al., 2005). Clavulanic acid-inhibited ESBLs conferring resistance to expanded spectrum cephalosporins have been reported, first in Enterobacteriaceae and then in Pseudomonas aeruginosa (Nordmann and Guibert, 1998). The TEMtype ESBLs are derivatives of TEM-1 and TEM-2. TEM-1 is able to hydrolyze ampicillin at a greater rate than carbenicillin, oxacillin, or cephalothin, and has negligible activity against extended- spectrum cephalosporins (Paterson and Bonomo, 2005). The SHV-type ESBLs may be more frequently found in clinical isolates than any other type of ESBLs (Jacoby, 1997). SHV refers to sulf hydryl variable. SHV-1 is a $\beta$-lactamase with activity against penicillins and narrow spectrum cephalosporins such as cephalothin and cephaloridine (Harada et al., 2008).

SHV-5 and SHV-12 are among the most common members of this family (Paterson et al., 2003). The most common group of ESBLs not belonging to the TEM or SHV families was termed CTX-M to highlight their greater activity against cefotaxime than against ceftazidime (Bonnet, 2004) but some of them, such as CTX-M-15 and -19 , also hydrolyse ceftazidime efficiently (Poirel et al., 2001) which may complicate their phenotypic recognition (Pitout et al., 2005). CTX-M ESBLs are the most commonly isolated ESBLs in many parts of the world, particularly Europe (Drawz et al., 2010). The OXA-type $\beta$ lactamases are so named because of their oxacillinhydrolyzing abilities. These $\beta$-lactamases are characterized by hydrolysis rates for cloxacillin and oxacillin greater than $50 \%$ that for benzylpenicillin (Paterson and Bonomo, 2005). GES-1 possesses hydrolytic activity similar to the classic class A ESBLs, is inhibited by $\beta$-lactamase inhibitors, and is generally classified into ESBLs (Poirel et al., 2000). However, some of the GES variants, such as GES-2 and 4, also have hydrolytic activity against carbapenems (Poirel and Weldhagen et al., 2001; Wachino et al., 2004). The occurrence and detection of ESBL in $P$. aeruginosa are undefined in Kashan. This study aimed to investigate the presence and prevalence of bla(TEM), bla(SHV), bla(CTX-M), bla(OXA) and bla(GES)-like genes, responsible for extended spectrum beta-lactamases (ESBLs) production, antibiotic susceptibilities and multidrug resistance (MDR) of $P$. aeruginosa, isolated from clinical specimens of patients of hospital.

\section{MATERIALS AND METHODS}

This descriptive study was carried out in Beheshti hospital from 2010 to 2011 (Kashan city, Isfahan province, Iran). 86 isolates of
$P$. aeruginosa were isolated from patients enrolled in this study. Conventional biochemical tests were used for identification at the species level in $86 \mathrm{~g}$ negative, short rods was based on colonial morphology, oxidase positivity, and the presence of characteristic pigments. The organisms were isolated from urine $(n=23)$, the trachea $(n=18)$, stool $(n=17)$, wound $(n=11)$, blood $(n=5)$, bronchoscopy fluid $(n=4)$, sputum $(n=3)$, vaginal discharge $(n=$ 2), pleural fluid $(n=1)$, gastrointestinal fluid $(n=1)$ and pus $(n=1)$. All isolates were cultured in Luria-Bertani (LB) broth and grown at $37^{\circ} \mathrm{C}$ for genomic DNA isolation. The isolates were stored in vials containing LB broth with $50 \%$ glycerol at $-20^{\circ} \mathrm{C}$. The purity of the strains was checked before of analysis. The susceptiblities of the bacterial isolates to eight antimicrobial agents (imipenem $10 \mu \mathrm{g}$, ciprofloxacin $5 \mu \mathrm{g}$, ceftazidime $30 \mu \mathrm{g}$, ceftriaxone $30 \mu \mathrm{g}$, cefotaxime $30 \mu \mathrm{g}$, aztreonam $30 \mu \mathrm{g}$, piperacillin $100 \mu \mathrm{g}$ and gentamicin $10 \mu \mathrm{g}$; [Mast Group Ltd., Merseyside, U.K.] were determined by the disk diffusion method according to the criteria published by the Clinical and Laboratory Standards Institute(CLSI). P. aeruginosa ATCC 27853 was used as quality control in each susceptibility determination. Multidrug-resistant isolates were defined as those resistant to three or more classes of antipseudomonal agents (carbapenems, penicillins, cephalosporins, monobactams, fluoroquinolones and aminoglycosides). ESBL production in all the isolates was detected by double-disc synergy test as described by Jarlier et al. (1998). Briefly of $30 \mu \mathrm{g}$ each ceftazidime, ceftriaxone, cefotaxime and aztreonam disks were placed at a $20 \mathrm{~mm}$ (center-to-center) from an amoxicillin-clavulanate 20/10 $\mu \mathrm{g}$ disk (Mast Group Ltd., Merseyside, U.K.) on Mueller-Hinton agar(Merck, Germany) and incubated at $37^{\circ} \mathrm{C}$ for $16 \mathrm{~h}$. Enhancement of the zone of inhibition of the oxyimino B-lactam due to synergy of the clavulanate in the amoxicillin-clavulanate disk was considered as evidence of ESBLproduction. This criterion also fulfills the CLSI guidelines. This increase occurs because the clavulanic acid present in the amoxyclav disc inactivates the ESBL produced by the test organism.

Bacterial DNA was prepared by suspending one or two fresh colonies in $100 \mathrm{ml}$ of sterile distilled water and heating at $95^{\circ} \mathrm{C}$ for $10 \mathrm{~min}$. After centrifugation, the supernatant was stored at $4^{\circ} \mathrm{C}$ before analysis. The detection of $\beta$-lactamase genes were performed under standard PCR conditions using published set of primers (Table 1). Amplicons were revealed by electrophoresis on a $1.2 \%$ agarose gel and a subsequent exposure to UV light in the presence of ethidium bromide.

\section{RESULTS}

$P$. aeruginosa were isolated from 86 patients. $P$. aeruginosa demonstrated the highest resistance rate to piperacillin $(38.4 \%)$, cefotaxime $(30.2 \%)$, imipenem $(30.2 \%)$, ceftriaxone $(25.6 \%)$, gentamicin (24.4\%), ceftazidime (20.9\%), aztreonam (19.8\%) and ciprofloxacin (17.4\%) respectively (Table 2 ). Nineteen $(20.9 \%) P$. aeruginosa isolates were resistant to all extended-spectrum cephalosporins tested. Among the total isolates, $7 \%$ were susceptible to all studied agents, and $8.1 \%$ were resistant to a single agent, predominantly to piperacillin (Table 3). Of the 86 isolates, 28 (32.5\%) were multi-drug resistant. From the total MDR strains, $89.3 \%$ were resistant to penicillins and $71.4 \%$ were resistant to the aminoglycosides. Of the majority of MDR isolates $(32.1 \%)$ were resistant to seven antimicrobial agents, and this group accounted for $10.5 \%$ of all isolates. Using the double-disk synergy test, ESBL- 
Table 1. Primers used in this study.

\begin{tabular}{|c|c|c|c|}
\hline Gene type & Primer & Sequences ( $5^{\prime}$ to $\left.3^{\prime}\right)$ & Product size (bp) \\
\hline \multirow[t]{2}{*}{ TEM-1 } & TEM-1 A & ATAAAATTCTTGAAGACGAAA & 1080 \\
\hline & TEM-1 B & GACAGTTACCAATGCTTAATCA & \\
\hline \multirow[t]{2}{*}{ SHV-1 } & SHV-1 A & TGGTTATGCGTTATATTCGCC & 865 \\
\hline & SHV-1 B & GGTTAGCGTTGCCAGTGCT & \\
\hline \multirow[t]{2}{*}{ SHV-5 } & SHV-5 A & ACTGAATGAGGCGCTTCC & 220 \\
\hline & SHV-5 B & CGCACCCCGCTTGCT & \\
\hline \multirow[t]{2}{*}{ OXA-1 } & OXA-1 A & AGCCGTTAAAATTAAGCCC & 908 \\
\hline & OXA-1 B & CTTGATTGAAGGGTTGGGCG & \\
\hline \multirow[t]{2}{*}{ CTX-M-1 } & CTX-M-1 A & GCGATGTGCAGCACCAGTAA & 605 \\
\hline & CTX-M-1 B & GGTTGAGGCTGGGTGAAGTA & \\
\hline \multirow[t]{2}{*}{ CTX-M-2 } & CTX-M-2 A & CGGAATTCATGATGACTCAGAGCATTCG & 605 \\
\hline & CTX-M-2 B & GCTCTAGATTATTGCATCAGAAACCGTG & \\
\hline \multirow[t]{2}{*}{ CTX-M-3 } & CTX-M-3 A & GGTTAAAAAATCACTGCG & 873 \\
\hline & CTX-M-3 B & TTACAAACCGTCGGTGA & \\
\hline \multirow[t]{2}{*}{ CTX-M-9 } & CTX-M-9 A & TATTGGGAGTTTGAGATGGT & 932 \\
\hline & CTX-M-9 B & TCCTTCAACTCAGCAAAAGT & \\
\hline \multirow[t]{2}{*}{ GES-1 } & GES-1 A & ATGCGCTTCATTCACGCAC & 860 \\
\hline & GES-1 B & CTATTTGTCCGTGCTCAGG & \\
\hline \multirow[t]{2}{*}{ GES-2 } & GES-2 A & GTTTTGCAATGTGCTCAACG & 371 \\
\hline & GES-2 B & TGCCATAGCAATAGGCGTAG & \\
\hline
\end{tabular}

Table 2. Antimicrobial susceptibility pattern on $P$. aeruginosa isolated from patients of Shahid Beheshti hospital.

\begin{tabular}{lccccc}
\hline $\begin{array}{l}\text { Antimicrobial } \\
\text { agents }(\boldsymbol{\mu g})\end{array}$ & $\begin{array}{c}\text { Resistant } \\
\text { isolates (\%) }\end{array}$ & $\begin{array}{c}\text { Intermediate } \\
\text { isolates }(\%)\end{array}$ & $\begin{array}{c}\text { Susceptible } \\
\text { isolates (\%) }\end{array}$ & $\begin{array}{c}\text { MDR isolates } \\
\text { NO. 28 (\%) }\end{array}$ & $\begin{array}{c}\text { ESBLs producing } \\
\text { isolates NO. 7 (\%) }\end{array}$ \\
\hline Piperacillin(100) & 38.4 & 0 & 61.6 & 89.3 & 71.4 \\
Cefotaxime (30) & 30.2 & 55.8 & 14 & 82.1 & 57.1 \\
Imipenem (10) & 30.2 & 2.3 & 67.5 & 89.3 & 42.9 \\
Ceftriaxone (30) & 25.6 & 13.9 & 60.5 & 71.4 & 57.1 \\
Gentamicin(10) & 24.4 & 2.3 & 73.3 & 71.4 & 42.9 \\
Ceftazidime 30) & 20.9 & 7 & 72.1 & 57.1 & 28.5 \\
Aztreonam (30) & 19.8 & 11.6 & 68.6 & 50 & 42.9 \\
Ciprofloxacin (5) & 17.4 & 11.6 & 71 & 53.6 & 14.3 \\
\hline
\end{tabular}

producing isolates were detected in only seven of the isolates. Maximum ESBL production was found in trachea and urine samples. Of the seven ESBL-positive isolates, three strains were multi-drug resistant.

PCR was carried out on the genomic DNA of seven
ESBL-positive isolates using primers for the following ESBL-encoding genes: bla $a_{\mathrm{TEM}-1}, b_{\mathrm{SHV}-1}, b / a_{\mathrm{SHV}-5}, b / a_{\mathrm{OXA}}$

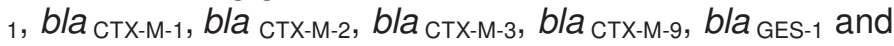
bla GES-2. Four of the ten genes were found alone or in various combinations. Seven strains were positive for 
Table 3. Resistance to one or more antimicrobials among $P$. aeruginosa isolated from patients of Shahid Beheshti hospital in Kashan.

\begin{tabular}{|c|c|c|c|c|c|c|c|c|c|}
\hline $\begin{array}{l}\text { No. of agents to } \\
\text { which isolates } \\
\text { were resistant }\end{array}$ & $\begin{array}{l}\text { Number and total } \\
\text { percent of isolates }\end{array}$ & $\begin{array}{l}\text { piperacillin } \\
\text { No. (\%) }\end{array}$ & $\begin{array}{l}\text { imipenem } \\
\text { No. (\%) }\end{array}$ & $\begin{array}{l}\text { gentamicin } \\
\text { No. (\%) }\end{array}$ & $\begin{array}{l}\text { Ciprofloxacin } \\
\text { No. (\%) }\end{array}$ & $\begin{array}{l}\text { ceftazidime } \\
\text { No. (\%) }\end{array}$ & $\begin{array}{l}\text { cefotaxime } \\
\text { No. (\%) }\end{array}$ & $\begin{array}{l}\text { ceftriaxone } \\
\text { No. (\%) }\end{array}$ & $\begin{array}{l}\text { aztreonam } \\
\text { No. (\%) }\end{array}$ \\
\hline 0 & $46(53.5)$ & & & & & & & & \\
\hline 1 & $7(8.1)$ & $6(85.7)$ & $1(14.3)$ & & & & & & \\
\hline 2 & $5(5.8)$ & $2(40)$ & & & & $2(40)$ & $3(60)$ & $2(40)$ & $3(50)$ \\
\hline 3 * & $5(5.8)$ & $2(40)$ & $5(100)$ & $3(60)$ & $3(60)$ & & $1(20)$ & & $1(20)$ \\
\hline $4^{*}$ & $2(2.3)$ & $2(100)$ & $2(100)$ & $1(50)$ & $1(50)$ & $1(50)$ & $1(50)$ & & \\
\hline 5 * & $5(5.8)$ & $5(100)$ & $3(60)$ & $2(40)$ & $3(60)$ & $2(40)$ & $5(100)$ & $4(80)$ & $1(20)$ \\
\hline 6 * & $4(4.6)$ & $4(100)$ & $3(75)$ & $4(100)$ & $3(75)$ & $1(25)$ & $4(100)$ & $4(100)$ & $1(25)$ \\
\hline $7^{*}$ & $9(10.5)$ & $9(100)$ & $9(100)$ & $8(88.9)$ & $2(22.2)$ & $9(100)$ & $9(100)$ & $9(100)$ & $8(88.9)$ \\
\hline $8^{*}$ & $3(3.5)$ & $3(100)$ & $3(100)$ & $3(100)$ & $3(100)$ & $3(100)$ & $3(100)$ & $3(100)$ & $3(100)$ \\
\hline
\end{tabular}

*28 out of $86(32.5 \%)$ isolates were resistant to three or more antimicrobials and defined as MDR.

bla GES-2 gene. One of seven $P$. aeruginosa isolates had the bla $\mathrm{SHV}_{-1}$ and bla $\mathrm{SHV}-5$ genes. Only one isolate was found to harbor the bla CTX-M-1 gene. However, none of the strains was positive

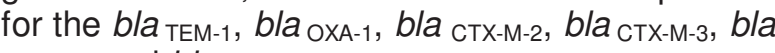
CTX-M-9 and bla $a_{\mathrm{GES}-1}$ genes.

\section{DISCUSSION}

The occurrence of multidrug-resistant $P$ aeruginosa strains is increasing worldwide and limiting our therapeutic options (Bonomo and Szabo, 2006). Our study reported the highest resistance rate to piperacillin, cefotaxime, imipenem, ceftriaxone, gentamicin, ceftazidime, aztreonam, ciprofloxacin respectively. In our study, gentamicin resistance value was higher than values reported in study conducted by Zhanel et al. (2010). In this study, ciprofloxacin exhibited nearly high susceptibility pattern $(71 \%)$ and Zhanel et al. (2010) reported that 19.0 to $24.1 \%$ isolates were resistant to fluoroquinolones.
Cephalosporins, are known anti-pseudomonal drugs, especially the third-genetration ceftadizime, that has demonstrated high susceptibility pattern $(72.1 \%)$ with $P$. aeruginosa isolates, however cefotaxime and ceftriaxone were the two of the cephalosporin drug tested in this study, with susceptibility value of 14 and $60.5 \%$ respectively. These low resistance value observed were comparable with the report from Malaysia of 40 and $31 \%$ (Jombo et al., 2008). In our study, prevalence of MDR $P$. aeruginosa isolates, was $32.5 \%$ which lower than values reported in other studies in Iran (Mirsalehian et al., 2010), Malaysia (Lim et al., 2009) and Pakistan (Ullah et al., 2009) and this rate is lower than study was performed in this hospital five years ago (Moniri et al., 2005). In contrast, Tam et al. (2010) reported that $14.0 \%$ of $P$. aeruginosa isolates were MDR. The multidrugresistant phenotype in $P$. aeruginosa could be mediated by several mechanisms including multidrug efflux systems, enzyme production, outer membrane protein (porin) loss and target mutations. Inappropriate empirical therapy has been associated with increased mortality in $P$. aeruginosa infections; delays in starting appropriate therapy may contribute to increased length of hospital stay and persistence of infection. In addition, worse clinical outcomes may be associated with MDR infections owing to limited effective antimicrobial options. Our study reported $8.1 \%$ ESBL production among $P$. aeruginosa isolates. The studies conducted by others depicted low rates, 3.7\% (Woodford et al., 2008), 4.2\% (Lim et al., 2009), 7.7\% (Jacobson et al., 1995) respectively, of ESBL production in $P$. aeruginosa. The results of various studies, showed high rate $20.3 \%$ (Aggarwal et al., 2008), 39.41\% (Mirsalehian et al., 2010) and 35.85\% (Ullah et al., 2009) of ESBLs in the samples of $P$. aeruginosa isolates examined. The other genes bla TEM-1, bla oXA-1, bla CTX-M-2, bla CTX-M-3, bla CTX-M-9 $_{\text {and }}$ anda $a_{\text {GES-1 }}$ genes were recently reported in $P$. aeruginosa were not detected. The first report of the SHV-1 penicillinase in epidemic $P$. aeruginosa strain showed by Kalai Blagui et al., 2009. Seven $P$. aeruginosa isolates producing the clavulanic 
acid-inhibited extended-spectrum beta-lactamase SHV-5 were isolated in the hospital in Athens, Greece (Poirel et al., 2004).

In our study, the bla $a_{\mathrm{GES}-2}$ gene was detected in all ESBL producing isolate. This was not accordance with other results in Middle East and other study in Iran. Recent reports from Brazil showed that bla $a_{\mathrm{GES}-1}$ are present in $P$. aeruginosa strains (Castanheira et al., 2004). In this study, we describe the presence of CTX-M-1 stlactamase in 1 clinical bacterial strains of $P$. aeruginosa. The first description of the presence of CTX-M ESBLs in $P$. aeruginosa and $S$. maltophili reported by $\mathrm{Al}$ Naiemi et al. (2006). The CTX-M ESBLs provide $P$. aeruginosa with an additional powerful resistance mechanism with potential serious clinical implications, including limitation of the therapeutic options. ESBLs producing organisms pose unique challenges to clinical microbiologists, clinicians, infection control professionals and scientists engaged in finding new antibacterial agents. The incidence of ESBLs varies with geographic location and time. ESBL producing strains are usually found in those areas of hospitals where antibiotic use is frequent and the patient's condition is critical. These resistant organisms are clinically important because they result in increased morbidity and mortality. In addition, ESBL producing bacteria are frequently resistant to many classes of antibiotics, resulting in difficult-to-treat infections. Currently, carbapenems are regarded as the drugs of choice for treatment of infections caused by ESBL-producing organisms. Unfortunately, use of carbapenems has been associated with the emergence of carbapenem-resistant Pseudomonas spp. The development and spread of ESBLs is most likely caused by the overuse of expandedspectrum cephalosporins in the hospital setting. Proper infection control practices and barriers are essential to prevent spreading and outbreaks of ESBL-producing bacteria.

In conclusion, gentamicin still shows the highest potency against $P$. aeruginosa in our hospital. However, emerging of resistance to carbapenems, in particular resistance to imipenem, may limit their prescription in the future. The prevalence of ESBL-producing $P$. aeruginosa occurred in $8.1 \%$ of isolates and MDR phenotype was common in $P$. aeruginosa. The most prevalent genes among ESBL producers were bla GES-2, which compromises the efficacy of imipenem, tend to be geographically restricted.

\section{ACKNOWLEDGMENTS}

This project was supported by Kashan University of Medical Sciences.

\section{REFERENCES}

Aggarwal R, Chaudhary U, Bala K (2008). Detection of extendedspectrum $\beta$ - lactamase in Pseudomonas aeruginosa. Indian. J.
Pathol. Microbiol., 51: 222-224.

Al Naiemi N, Duim B, Bart A (2006). A CTX-M extended-spectrum betalactamase in Pseudomonas aeruginosa and Stenotrophomonas maltophilia. J. Med. Microbiol., 55: 1607-1608.

Bonnet $R$ (2004). Growing group of extendedspectrum $b$-lactamases: the CTX-M enzymes. Antimicrob Agents Chemother., 2004 Jan; 48(1): $1-14$

Bonomo RA, Szabo D (2006). Mechanisms of multidrug resistance in Acinetobacter species and Pseudomonas aeruginosa. Clin. Infect. Dis., 1(43) Suppl 2: S49-56.

Bradford PA (2001). Extended-spectrum b-lactamases in the 21st century: characterization, epidemiology, and detection of this important resistance threat. Clin. Microbiol. Rev., 14(4): 933-51.

Castanheira M, Mendes RE, Walsh TR, Gales AC, Jones RN (2004). Emergence of the extended-spectrum s-lactamase GES-1 in a Pseudomonas aeruginosa strain from Brazil: report from the SENTRY antimicrobial surveillance program. Antimicrob. Agents. Chemother., 48(6): 2344-2345.

Drawz SM, Bonomo RA (2010). Three Decades of $\beta$-Lactamase Inhibitors. Clin. Microbiol. Rev., 23: 160-201.

Dundar D, Otkun M (2010). In-Vitro Efficacy of Synergistic Antibiotic Combinations in Multidrug Resistant Pseudomonas Aeruginosa Strains. Yonsei. Med. J., 51: 111-116.

Harada S, Ishii Y, Yamaguchi K (2008). Extended-spectrum betalactamases: implications for the clinical laboratory and therapy. Korean. J. Lab. Med., 28: 401-412.

Jacoby GA (1997). Extended-spectrum beta-lactamases and other enzymes providing resistance to oxyimino-beta-lactams. Infect. Dis. Clin. North. Am., 11: 875-887.

Jarlier V, Nicolas MH, Fournier G, Philippon A (1998). Extended spectrum $\beta$-lactamases conferring transferable resistance to newer $\beta$ lactam agents in Enterobacteriaceae: Hospital prevalence and susceptibility patterns. Rev. Infect. Dis., 10: 867-878.

Jombo GT, Jonah P, Ayeni JA (2008). Multidrug Resistant Pseudomonas aeruginosa in Contemporary Medical Practice: Findings From Urinary Isolates At A Nigerian University Teaching Hospital. Niger. J. Physiol. Sci., 23: 105-109.

Kalai Blagui S, Achour W, Bejaoui M, Abdeladhim A, Ben Hassen A (2009). Detection of SHV-1 beta-lactamase in Pseudomonas aeruginosa strains by genetic methods. Pathol. Biol. (Paris)., 57: 7375.

Lim KT, Yasin RM, Yeo CC, Puthucheary SD, Balan G, Maning N, Wahab ZA, Ismail N, Tan EA, Mustaffa A, Thong KL (2009). Genetic fingerprinting and antimicrobial susceptibility profiles of Pseudomonas aeroginosa hospital isolates in Malaysia. J. Microbiol. Immunol. Infect., 42: 197-209.

Mirsalehian A, Feizabadi M, Nakhjavani FA, Jabalameli F, Goli $H$, Kalantari N (2010). Detection of VEB-1, OXA-10 and PER-1 genotypes in extended-spectrum beta-lactamase-producing Pseudomonas aeruginosa strains isolated from burn patients. Burns, 36: 70-74.

Moniri R, Mosayebi Z, Movahedian AH, Mussavi GA (2006). Increasing Trend of Antimicrobial Drug-Resistance in Pseudomonas aeruginosa Causing Septicemia. Iranian J. Publ. Health., 35: 58-62.

Nordmann P, Guibert M (1998). Extended-spectrum beta-lactamases in Pseudomonas aeruginosa. J. Antimicrob Chemother., 42:128-131.

Paterson DL, Bonomo RA (2005). Extended-Spectrum $\beta$-Lactamases: a Clinical Update. Clin. Microbiol. Rev., 18: 657-686.

Paterson DL, Hujer KM, Hujer AM, Yeiser B, Bonomo MD, Rice LB, Bonomo RA (2003). Extended-spectrum beta-lactamases in Klebsiella pneumoniae bloodstream isolates from seven countries: dominance and widespread prevalence of SHV- and CTX-M-type beta-lactamases. Antimicrob. Agents. Chemother., 47: 3554-3560.

Pitout JD, Nordmann P, Laupland KB, Poirel L (2005). Emergence of Enterobacteriaceae producing extended-spectrum beta-lactamases (ESBLs) in the community. J. Antimicrob. Chemother., 53: 52-59.

Poirel L, Naas T, Le Thomas I, Karim A, Bingen E, Nordmann P (2001). CTX-M-type extendedspectrum b-lactamase that hydrolyzes ceftazidime through a single amino acid substitution in the omega loop. Antimicrob. Agents. Chemother., 45: 3355-3361.

Poirel L, Le Thomas I, Naas T, Karim A, Nordmann P (2000). Biochemical sequence analyses of GES-1, a novel class $A$ extended- 
spectrum $\beta$ - lactamase, and the class 1 integron In52 from Klebsiella pneumoniae. Antimicrob. Agents. Chemother., 44: 622-632.

Poirel L, Weldhagen GF, Naas T, De Champs C, Dove MG, Nordmann $P$ (2001). GES-2, a class A $\beta$-lactamase from Pseudomonas aeruginosa with increased hydrolysis of imipenem. Antimicrob. Agents. Chemother., 45: 2598-2603.

Rossolini GM, Mantengoli E (2005). Treatment and control of severe infections caused by multiresistant Pseudomonas aeruginosa. Clin. Microbiol. Infect., 11: 17-32.

Tam VH, Chang KT, Abdelraouf K, Brioso CG, Ameka M, McCaskey LA, Weston JS, Caeiro JP, Garey KW( 2010). Prevalence, Resistance Mechanisms, and Susceptibility of Multidrug-Resistant Bloodstream Isolates of Pseudomonas aeruginosa. Antimicrob. Agents. Chemother., 54: 1160-1164.

Ullah F, Malik SA, Ahmed J (2009). Antimicrobial susceptibility and ESBL prevalence in Pseudomonas aeruginosa isolated from burn patients in the North West of Pakistan. Burns, 35(7): 1020-5

Wachino J, Doi Y, Yamane K, Shibata N, Yagi T, Kubota T, Arakawa Y (2004). Molecular characterization of a cephamycin-hydrolyzing and inhibitor- resistant class A $\beta$ lactamase, GES-4, possessing a single G170S substitution in the omega-loop. Antimicrob Agents Chemother., 48: 2905-2910.
Wirth FW, Picoli SU, Cantarelli VV, Gonçalves AL, Brust FR, Santos LM, Barreto MF (2009). Metallo- $\beta$-lactamase-producing Pseudomonas aeruginosa in two hospitals from Southern Brazil. Braz. J. Infect. Dis., 13: 170-172.

Woodford N, Zhang J, Kaufmann ME, Yarde S, Tomas Mdel M, Faris C, Vardhan MS, Dawson S, Cotterill SL, Livermore DM (2008). Detection of Pseudomonas aeruginosa isolates producing VEB-type extended-spectrum $\beta$-lactamases in the United Kingdom. J. Antimicrob. Chemother., 62: 1265-68.

Zhanel GG, DeCorby M, Adam H, Mulvey MR, McCracken M, LagacéWiens P, Nichol KA, Wierzbowski A, Baudry PJ, Tailor F, Karlowsky JA, Walkty A, Schweizer F, Johnson J; Canadian Antimicrobial Resistance Alliance, Hoban DJ (2010). Prevalence of antimicrobialresistant pathogens in Canadian hospitals: results of the Canadian Ward Surveillance Study (CANWARD 2008). Antimicrob. Agents. Chemother., 54(11): 4684-4693. 